\title{
Prenatal diagnosis of dwarfism by ultrasound screening
}

\author{
B-M WELDNER, P-H PERSSON, AND S A IVARSSON \\ Departments of Obstetrics and Gynaecology and Paediatrics, University of Lund, General Hospital, \\ Malmö, Sweden
}

SUMMARY In a general, ultrasound screening programme, 12453 women were examined at 16 and 32 weeks of pregnancy. The screening detected all limb deformities in the population during the study period. The seeming prevalence of dwarfism in the population was 750 per million.

Since 1973, almost all pregnant women $(97 \%)$ in Malmö have undergone routine ultrasound examination in conjunction with a screening programme introduced to improve the diagnosis of multiple pregnancy. It soon became evident that the major improvement due to routine screening was the objective assessment of gestational age by biparietal diameter measurement in the first half of pregnancy. At present one examination to assess gestational age is performed at 16 weeks, and another is undertaken at 32 weeks to judge fetal growth. Besides fetometry, fetal anatomy is carefully studied, structural abnormalities being detected in $0.7 \%$ of the examinations.

During the five year period 1 January 1980 to 31 March 1985, 12453 pregnant women were examined. The examinations were performed by two specially trained midwives. At each examination, fetal arms and legs were examined and counted, but only femur length was objectively measured (according to O'Brian). ${ }^{1}$ Normal values of femur length for gestational age were established in this hospital in 1979 (unpublished data).

The Department of Obstetrics and Gynaecology in Malmö is the sole maternity department serving a city of 240000 inhabitants. All deliveries in the area take place at one maternity unit. No external referrals have been included in the study.

\section{Results}

The results regarding limb deformities are summarised in the Table. At the 16 week examination, six cases of limb deformity were diagnosed. Three fetuses had extremely short limbs, two of them also had narrow thoracic cages. Reduced echogenicity of the spine was detected in two fetuses. In one of them, the arms were of normal length and the thoracic cage seemed normal on ultrasound, but the femurs were short, and both tibia and fibula severely angulated. In the other, arms and legs were too short and the thorax seemed narrow. These two pregnancies were terminated by hysterotomy and at necropsy the fetus with angulated legs was found to have multiple fractures of the legs and was diagnosed as a case of osteogenesis imperfecta, while the fetus with the narrow thoracic cage was also found to have a cleft palate and was classified as achondrogenesis type 1 . Also at necropsy, two cases of thanatophoric dwarfism (with narrow thoracic cages) and one case of Ellis Van Creveld syndrome (with normal thoracic cage) were found. In one case, fetal anatomy seemed normal, though the lower limbs were short and measured only $14 \mathrm{~mm}, 6$ $\mathrm{mm}$ below expected value for gestational age and biparietal diameter. The mother had diabetes mellitus. The diagnosis of caudal regression syndrome was confirmed at necropsy.

At 32 weeks, two pregnancies were found where

Table Results of the ultrasound screening with regard to detection of limb deformities

\begin{tabular}{|c|c|c|c|c|}
\hline No & $\begin{array}{l}\text { Week of } \\
\text { detection }\end{array}$ & Fetal ultrasound finding & Diagnosis & Heredity \\
\hline 2 & 16 & Extremely short limbs, narrow thorax, normal echogenicity of the spine & Thanatophoric dwarfism & - \\
\hline 1 & 13 & Extremely short limbs, normal thorax, normal echogenicity of the spine & Ellis van Crefeld syndrome & - \\
\hline 1 & 16 & $\begin{array}{l}\text { Reduced echogenicity of the spine, normal thorax, angulated short femurs, } \\
\text { slightly reduced length of upper }\end{array}$ & Osteogenesis imperfecta & - \\
\hline 1 & 16 & Reduced echogenicity of the spine, narrow thorax, cleft palate, very short limbs & Achondrogenesis type 1 & - \\
\hline 1 & 16 & Moderately short limbs, femurs below normal, mean -4 SD & Caudal regression syndrome & $\begin{array}{l}\text { Diabetes } \\
\text { mellitus }\end{array}$ \\
\hline 1 & 32 & $\begin{array}{l}\text { Short limbs }(<\text { mean }-4 \mathrm{SD}) \text { normal at } 16 \text { weeks, normal echogenicity of } \\
\text { the spine, no other malformations }\end{array}$ & Achondroplasia & - \\
\hline 1 & 33 & Short lower extremities $(<$ mean $-3 \mathrm{SD})$ & Unknown, intrauterine death & - \\
\hline
\end{tabular}


the fetus had short limbs. In both cases, femur length had been measured at 16 weeks and found to be between the 50th and 75th centile according to our scale. The Figure shows the values for fetal biparietal diameter, mean abdominal diameter, and femur length for both of these fetuses in the third trimester. Femur lengths were below -4 to $-5 \mathrm{SD}$, but both biparietal and abdominal diameters were within the normal range. One of these two fetuses, an achondroplastic heterozygote, weighed $3.260 \mathrm{~g}$ when delivered at term; the other, which had no noticeable defects other than the pronouncedly short limbs, weighed $3.120 \mathrm{~g}$ when it died in utero at 38 weeks.

Six of seven cases detected seemed to be mutations as no familial history could be found. The
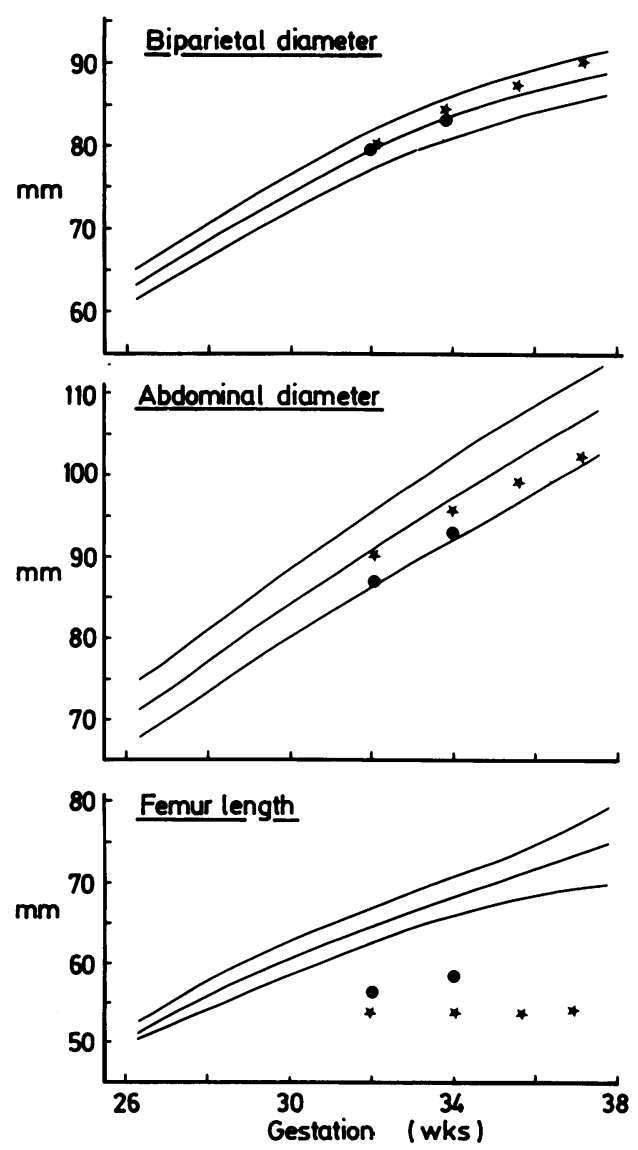

Figure Measurements at 32 weeks-biparietal diameter, mean abdominal diameter, and femur length for two dwarfs whose femurs had been of normal length at 16 weeks of gestation. mother whose fetus had Ellis Van Creveld syndrome had previously had one normal delivery and three early spontaneous abortions; her last pregnancy (after that reported here) also had to be terminated owing to limb deformity in the fetus, discovered with ultrasound during the 11th week (this case is not reported here).

\section{Discussion}

Our findings suggest a frequency for limb deformity of 750 per million pregnancies. Many studies on prenatal diagnosis have indicated that the frequency of disease in utero is higher than the prevalence of disease at delivery. In 1971, Harris and Patton ${ }^{2}$ reported the incidence of achondroplasia and thanatophoric dwarfs at birth to be between 28 and 266 per million, which is slightly below the present finding.

The screening programme resulted in the detection of all limb deformities in the population during the study period. No false results were obtained. With regard to limb deformities, the screening had a sensitivity and predictive value of $100 \%$. Six of seven lethal malformations were unequivocally diagnosed at the 16 week examination. In five of six cases detected at 16 weeks, the deformities were severe and unmistakable. Direct measurement of the arms and legs was unnecessary to obtain diagnosis, except in the case of caudal regression syndrome, but was done to confirm diagnosis objectively. In most cases, the long bones were not only short but typically bowed and broadened too; the case of osteogenesis imperfecta being no exception to this sonographic pattern owing to multiple fractures and dislocations.

In the evaluation of a prenatal finding of short arms and legs, a judgement of the thoracic cage, abdomen, and facial skeleton should be made. The echogenicity of the fetal spine and the degree of reduction of upper/lower and distal/proximal arms and legs should be observed in order to obtain correct diagnosis. It is worth remembering that the heterozygous cases of achondroplasia had normal femur length measurements at 16 weeks. At 32 weeks, however, the femur lengths were approximately equal to 28 weeks of gestation, and in repeated examinations, no further growth of the femurs could be noticed. Filly and Golbus ${ }^{3}$ have previously reported that in cases of fetal achondroplasia, growth rates in homozygous fetuses differ from those in heterozygous fetuses. This is illustrated by our findings here, and it might serve to reassure those who are hesitant with regard to ultrasound in pregnancy on the grounds that otherwise healthy dwarfs might be aborted as a result of 
screening. The heterozygous forms are probably not detectable during the first half of pregnancy.

References

${ }^{1}$ O'Brian GD, Queenan JT, Campbell S. Assessment of gestational age in the second trimester by real-time ultrasound measurement in the femur length. Am J Obstet Gynecol 1981;139:340-5.

2 Harris R, Patton JT. Achondroplasia and thanatophoric dwarfism in the newborn. Clin Genet 1972:2:61-72.
${ }^{3}$ Filly RA, Golbus MS, Carey JC, Hall JG. Short-limbed dwarfism: ultrasonic diagnosis by mensuration of fetal femoral length. Radiology 1981;138:653-6.

Correspondence to Dr P-H Persson, Department of Obstetrics and Gynaecology, University of Lund, General Hospital, S-214 01 Malmö, Sweden.

Received 18 June 1985

\title{
Subependymal cysts in normal neonates
}

\author{
E-Y SHEN AND F-Y HUANG \\ Department of Paediatrics, Mackay Memorial Hospital, Taipei, Taiwan
}

SUMMARY We undertook a prospective ultrasound study of subependymal cyst formation in normal neonates. Twenty five of 500 normal Chinese neonates $(5 \%)$ were found to have subependymal cysts. Most of these (18 of 25) showed no obvious perinatal insult. This study suggests a rather high incidence of concealed intrauterine subependymal haemorrhage in normal term neonates.

Subependymal pseudocysts in a neonate suggest an intrauterine pathology. Larroche ${ }^{1}$ described these cystic formations, mainly located within the remnant zone germinativa or close to it, and considered that antenatal insults accounted for the cellular destruction in the remnant matrix. A germinal matrix haemorrhage may also develop into a subependymal cyst. $^{23}$

In this study, 25 of $500(5 \%)$ normal neonates were found to have subependymal cysts soon after their birth. These neonates were all healthy at birth and during scanning.

We suggest that subependymal haemorrhage may develop in term as well as preterm fetuses at the same gestational age and cause these cystic formations at birth.

\section{Materials and methods}

Between June and August 1984, 500 of 1701 $(29.4 \%)$ normal neonates in this hospital were scanned after obtaining informed parental consent. There were no differences in social class or economic status between the parents who gave or refused their consent. There were 265 boys and 235 girls, whose ages ranged from 2 to 9 days.
A commercially available, portable real time sector scanner with $5 \mathrm{MHz}$ rotatory head transducer and a $80^{\circ}$ field of vision was used at the bedside according to a standard method. ${ }^{3}$

Physical examination is performed routinely on every neonate at the age 2 or 3 days in this nursery. If a neonate was noted to have subependymal cyst, he or she was then assessed again physically and neurologically soon after scanning.

Patients were asked to come back for repeat ultrasound scans monthly until resolution of cysts.

\section{Results}

Subependymal cysts were discovered in $25(5 \%)$ of 500 neonates, $12(4.5 \%)$ of whom were boys and 13 $(5.5 \%)$ girls. Their gestational age ranged from 37 weeks to 43 weeks, mean 39.2 weeks (Fig. 1). Birthweight ranged from $2800 \mathrm{~g}$ to $4000 \mathrm{~g}$, mean $3302 \mathrm{~g}$ and occipitofrontal diameter ranged from 31 $\mathrm{cm}$ to $37 \mathrm{~cm}$, mean $33.6 \mathrm{~cm}$.

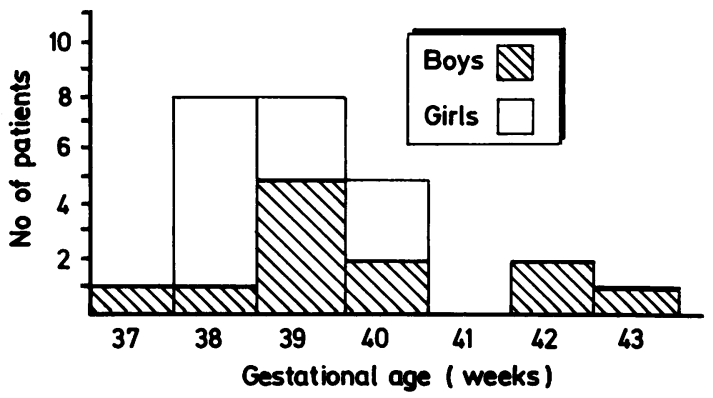

Fig. 1 Gestational age distribution in 25 neonates with subependymal pseudocysts. 